\title{
Effect of food and anti-cholinergic drugs on the pattern of rectosigmoid contractions
}

\author{
J Daly, A Bergin, W M Sun, N W Read
}

\begin{abstract}
The colonic response to a meal is often used to test the effect of drugs on colonic motility, but this test is hindered by its inconsistency. This study has used multiple manometric sensors situated in the rectosigmoid region to investigate whether recording of the site and type of contraction offers a clear discrimination of the colonic response to a meal and the effect of drugs. Two studies were carried out on 16 healthy volunteers. Before the meal, rectosigmoid motility consisted mainly of isolated contractions occurring in a single manometric channel. The motility index increased in every subject after the meal $(p<0.05)$, but this increase entirely consisted of a massive increase in contractions occurring simultaneously in three or more manometric channels (multiple channel contractions), the number increasing from 9 per hour preprandially to 57 per hour $(\mathrm{p}<0.01)$. There was a concomitant decrease in the number of the single channel contractions from 65 to 56 per hour. In a second study an infusion of an antispasmodic drug, mebeverine hydrochloride, into the sigmoid colon of healthy volunteers stopped the postprandial increase in the multiple channel contractions and prevented the signficiant rise in the motility index. The decrease in single channel contractions was unaffected. These results show that the colonic response to a meal consists of a change in the pattern of rectosigmoid contractions and suggest that multiple channel contractions may be a more sensitive indicator of the effect of a meal on the rectosigmoid colon than the motility index.
\end{abstract}

(Gut 1993; 34: 799-802)

One of the most widely reported aspects of colonic motility is the increase in colonic motor activity that occurs after a meal; the so-called gastrocolonic response. This response is said to be increased in patients with the irritable bowel syndrome' ${ }^{1}$ where it may be associated with feelings of imminent defecation and pain. ${ }^{2}$ Recordings of the gastrocolonic response are often used to test the action of drugs that reduce colonic motor activity and may be used to treat the irritable bowel syndrome.

A serious problem in using the gastrocolonic response to test the effect of drugs is its variability; being very obvious in some subjects but barely discernable in others. ${ }^{3}$ There are two main reasons for this variability; firstly, the large fluctuations in colonic activity under basal conditions that make changes related to meals difficult to detect ${ }^{4}$ and secondly, that increases in pressure activity may occur in a discrete high pressure zone some 12 to $15 \mathrm{~cm}$ from the anal verge $^{5}$ and may be missed if only single or tandem sensors are used.

\section{Subjects}

There were a total of 16 healthy male volunteers aged between 21 and 40 years, all were nonsmokers with no history of gastrointestinal disturbance. Each subject had a full medical examination and gave written informed consent before taking part in the study.

\section{Method}

Two studies were carried out. In the first, conducted on eight volunteers, the effect of a meal on the pattern of rectosigmoid contractions was studied. In the second, also carried out on eight subjects, the effect of a local infusion of mebeverine hydrochloride on the gastrocolonic response to a meal was investigated.

Each subject had a bowel preparation before the manometric probe was inserted using a rigid sigmoidoscope. The manometric probe used was a six lumen polyvinyl tube (external diameter $4 \mathrm{~mm}$ ) and had side opening ports situated at 22 , $18,15,12$, and $1.5 \mathrm{~cm}$ from the anal verge when the tube was in situ. These pressure channels were perfused with sterile water at $0.33 \mathrm{ml} / \mathrm{min}$ by a low compliance pressurised perfusion system (Arndorfer Medical Specialities Inc, Wisconsin, USA) and connected by water filled pressure transducers (PDCR 75 Druck Ltd, Groby, Leicestershire) to an eight channel chart recorder (Grass Model 7D Polygraph).

PROTOCOL ONE - THE EFFECT OF A MEAL ON THE PATTERN OF RECTOSIGMOID CONTRACTIONS

For three days before the test each subject ate a low residue diet while maintaining a high fluid intake. Then on the day before each test, the bowel was cleared by taking two sachets of Picolax (sodium picosulphate $10 \mathrm{mg}$ per sachet), one on rising and one after lunch. Subjects then fasted overnight and arrived in the department at $9 \mathrm{am}$. They were invited to empty their bowels if they felt the need to do so before insertion of the manometric probe.

A 15 minute equilibration or rest period was allowed before the recording began. After three hours of basal recording, the subjects ate a high fat meal, which consisted of a quarter pound beefburger (Birdseye) in a wholemeal bun (Hovis), $20 \mathrm{~g}$ mayonnaise (Hellmans), and $66 \mathrm{~g}$ ice cream (Walls) and $200 \mathrm{ml}$ coffee and $20 \mathrm{ml}$ milk. This provided a meal of about $900 \mathrm{kcals}$ with the distribution of calories between protein, carbohydrate, and fat being $12 \cdot 5 \%, 31 \cdot 7 \%$, and $55 \cdot 8 \%$ respectively. The meal was consumed 
within 15 minutes by all subjects. Recordings were continued for a further two hours after ingestion of the meal.

PROTOCOL TWO - EFFECT OF A LOCAL INFUSION OF MEBEVERINE HYDROCHLORIDE ON THE GASTROCOLONIC RESPONSE

A double blind placebo controlled randomised single dose crossover study was carried out to study the effect of an infusion of $75 \mathrm{mg}$ of mebeverine hydrochloride into the sigmoid colon on rectal motility. The studies were carried out with a gap of three days between them.

The manometric probe was modified by the addition of an extra port which was situated at $21 \mathrm{~cm}$ from the anal verge, through the mebeverine hydrochloride or the placebo (saline) was introduced. On the morning of each test day, after an overnight fast, subjects were given bowel preparation two hours before the start of each test. This consisted of a low, warm water enema sufficient to clear the rectum of faeces. A 45 minute equilibration period was followed by basal recording for one hour. The test solution was then infused into the sigmoid colon and recording was continued for another 90 minutes. Then the high fat test meal was given and both the postprandial recording and infusion was continued for 60 minutes. During this study, the volunteers were asked to indicate the occurrence of any rectal sensations on the chart using a remote event marker.

\section{Measurements and analysis}

The motility index was verified by summing the areas under all of the rectal and sigmoid recordings to zero pressure baseline by means of an electronic digitising board (CalComp, Anaheim, California) connected to a computer (BBC).

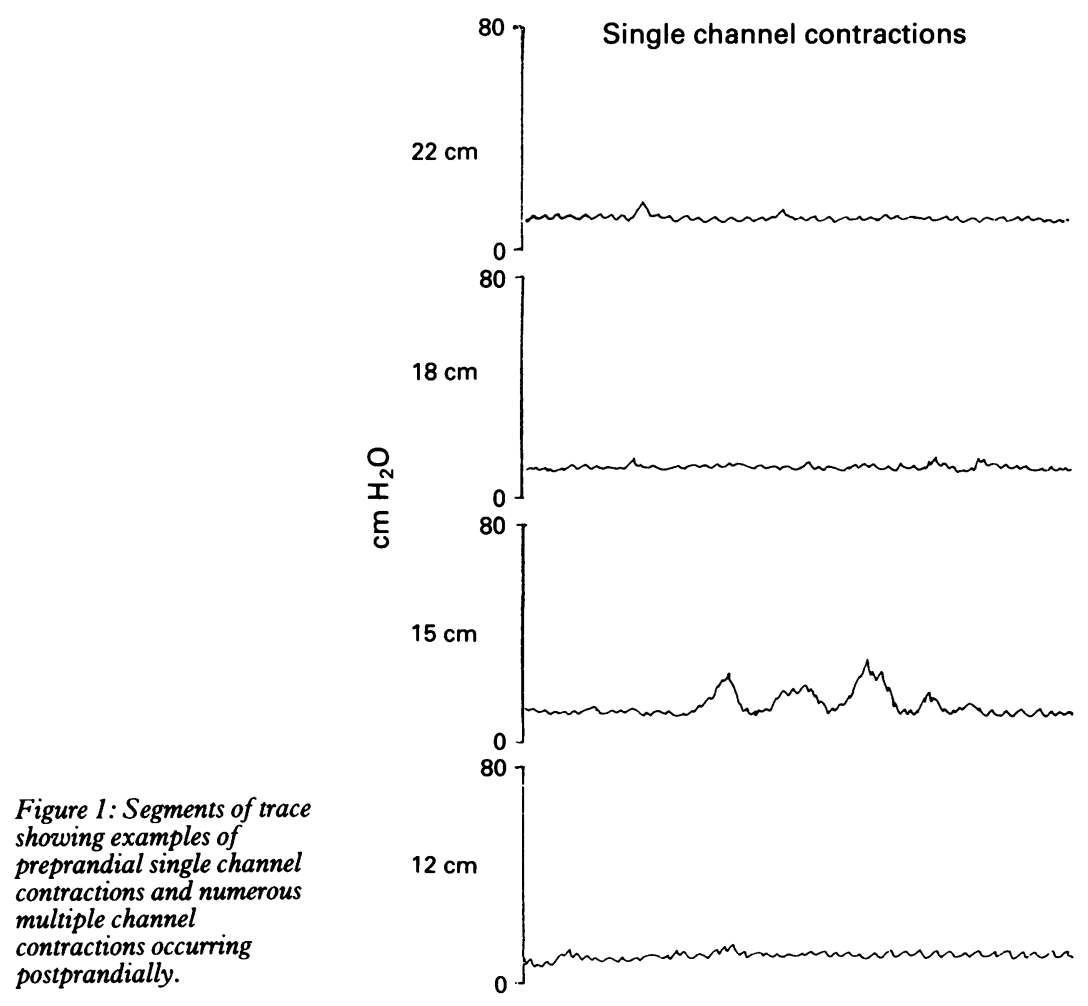

In addition, the number of contractions that occurred in just one channel were summed and expressed as the frequency of isolated or single channel contractions. A contraction was defined as a rise in pressure of at least $5 \mathrm{~cm}$ water with a duration of at least three seconds. Contractions that occurred in three or more adjacent channels simultaneously were counted as multiple channel contractions. To qualify as a multiple channel contraction the onset of pressure waves in adjacent channels had to occur within one second.

Artefacts as a result of movement, coughing, talking, etc, were distinguished from multiple channel contractions because they had more sharply peaked profiles, which were the same in all channels, including the anal channel. To facilitate identification of artefacts any movement of the subject was marked on the trace by the investigator during the study.

The data are expressed as motility index/hour and contraction frequency/hour for the pre and postprandial periods. Differences between preprandial and postprandial records and between placebo and mebeverine periods were determined using the Student's paired $t$ test with a significance value of $5 \%$.

\section{Results}

EFFECT OF A MEAL ON RECTOSIGMOID MOTILITY Before the meal there was no obvious pattern to the pressure activity recorded under basal or fasting conditions. When activity was seen, it usually occurred as single contractions or contraction clusters in a single channel (Fig 1). There was a wide intersubject variation with frequency of contractions ranging between 20 and 132 per hour for the single channel type and 0 and 20 per hour for the multiple channel contractions.

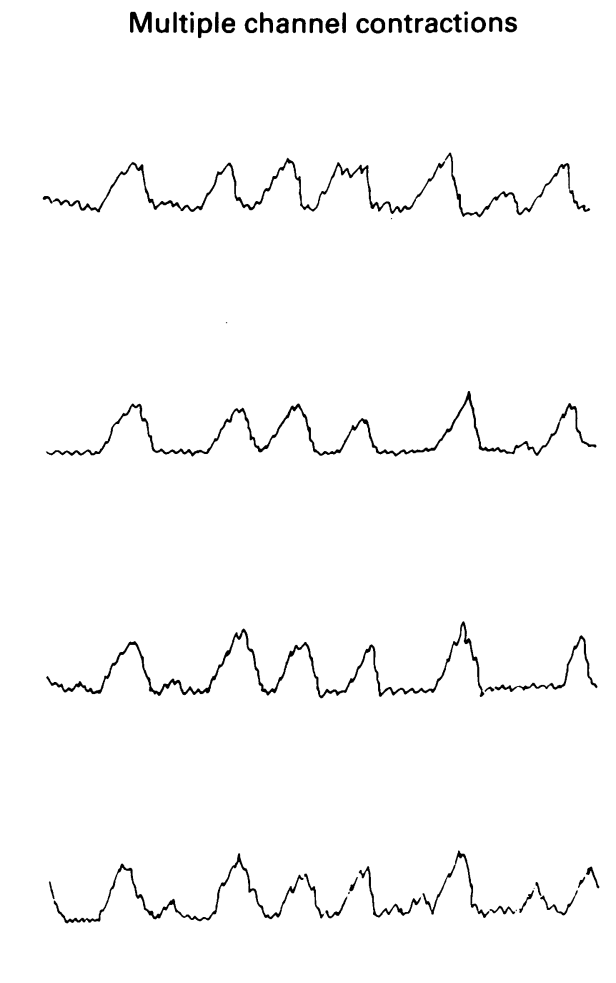

Figure 1: Segments of trace showing examples of preprandial single channel contractions and numerous multiple channel contractions occurring postprandially. 
TABLE I Effect of a meal on rectosigmoid motility

\begin{tabular}{lcc}
\hline & Preprandial & Postprandial \\
\hline Motility index & $17285(2297)$ & $298880(3090)^{\star}$ \\
Single channel contraction/h & $65(10)$ & $56(11)$ \\
Multiple channel contraction/h & $9(3)$ & $57(9) \dagger$ \\
\hline
\end{tabular}

Results show mean of eight subjects $($ SEM $) ;{ }^{\star}=\mathrm{p}<0.05$ significantly different from preprandial; $\dagger=p<0.01$ significantly different from preprandial.

After ingestion of the meal there was a significant increase in the motility index $(p<0.05)$ that occurred within 15 minutes after meal ingestion for each subject and remained raised for the next three hours (Table I, Fig 2). This increase in motility consisted mainly of a massive and highly significant $(p<0.01)$ increase in the number of multiple channel contractions which was seen in all subjects (range 28 to 100 per hour) (Fig 3). In contrast, there was a concomitant decrease in the number of a single channel contractions that also occurred in all of the subjects (Fig 3).

\section{EFFECTS OF LOCAL INFUSION OF MEBEVERINE HYDROCHLORIDE ON THE GASTROCOLONIC RESPONSE}

The basal numbers of multiple channel contractions in this study were greater than in the previous study, possibly as a result of the more active bowel preparation. Infusion of mebeverine did not significantly influence the preprandial recordings, but there were significant differences in the meal response between the placebo and mebeverine studies. During the placebo infusion there was a significant postprandial increase in the motility index $(p<0.05)$ caused by a significant $(p<0.05)$ rise in the frequency of the multiple channel contractions (Table II). This was accompanied by a decreased number of single channel contractions. During infusion of mebeverine there was not a statistically significant increase in the motility index, the rise seen was because of a tonic increase in the tone of the rectum which resulted in higher pressure overall. Mebeverine stopped the increase in multiple channel contractions but had no effect on single channel contractions, which decreased postprandially as before.

In five of eight subjects, all episodes of rectal sensation (desire to defecate, urgency or pain) were associated with multiple channel contractions. One subject experienced no sensations and in the remaining two subjects 9 of 32 and 8 or 19 sensory episodes were associated with multiple channel contractions.

TABLE II Effect of mebeverine hydrochloride on postprandial rectosigmoid motility

\begin{tabular}{lllll}
\hline & $\begin{array}{l}\text { Placebo } \\
\text { before }\end{array}$ & after & $\begin{array}{l}\text { Mebeverine } \\
\text { before }\end{array}$ & after \\
\hline Motility index & $28449(5292)$ & $48823(7966)^{\star}$ & $29916(6261)$ & $44407(7432)$ \\
Single channel contractions/h & $39(21)$ & $27(9)$ & $69(21)$ & $33(10)$ \\
Multiple channel contractions/h & $26(4)$ & $37(6)^{\star}$ & $21(5)$ & $21(6) \dagger$ \\
\hline
\end{tabular}

Results show mean of eight subjects $(\mathrm{SEM}) ;^{\star}=\mathrm{p}<0.01$ significantly different from preprandial; $\dagger=p<0.05$ significantly different from placebo.

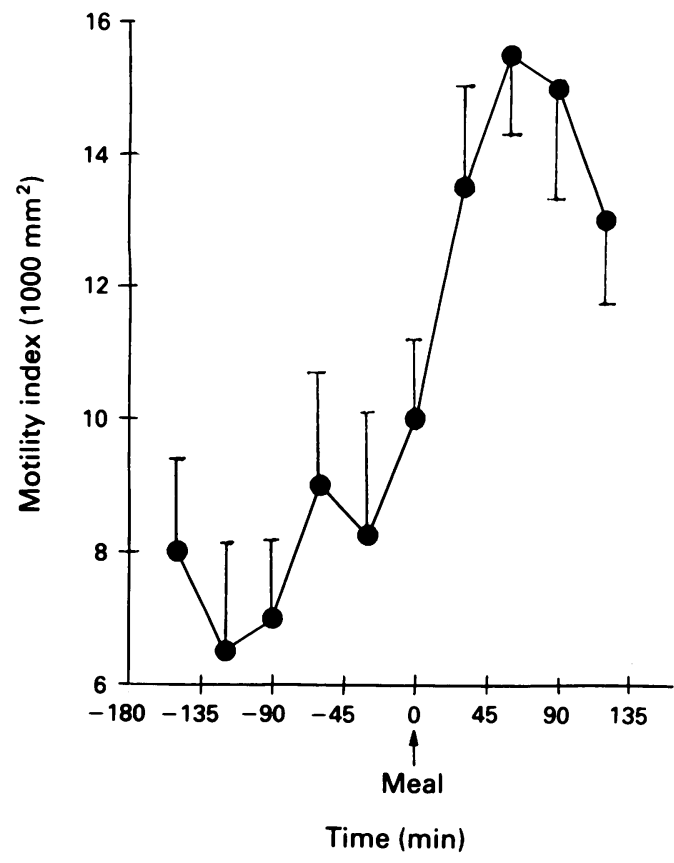

Figure 2: The mean motility index before and after a meal in study 1 .

\section{Discussion}

The results of the study show that the rectosigmoid motor response to a meal can be explained by a dramatic increase in contractions that occurred simultaneously in all rectosigmoid channels.

Before the meal, the manometric record consisted of sporadic contractions confined to a single channel, similar to the low amplitude nonpropulsive segmental contractions seen by Narducci and his colleagues. ${ }^{6}$

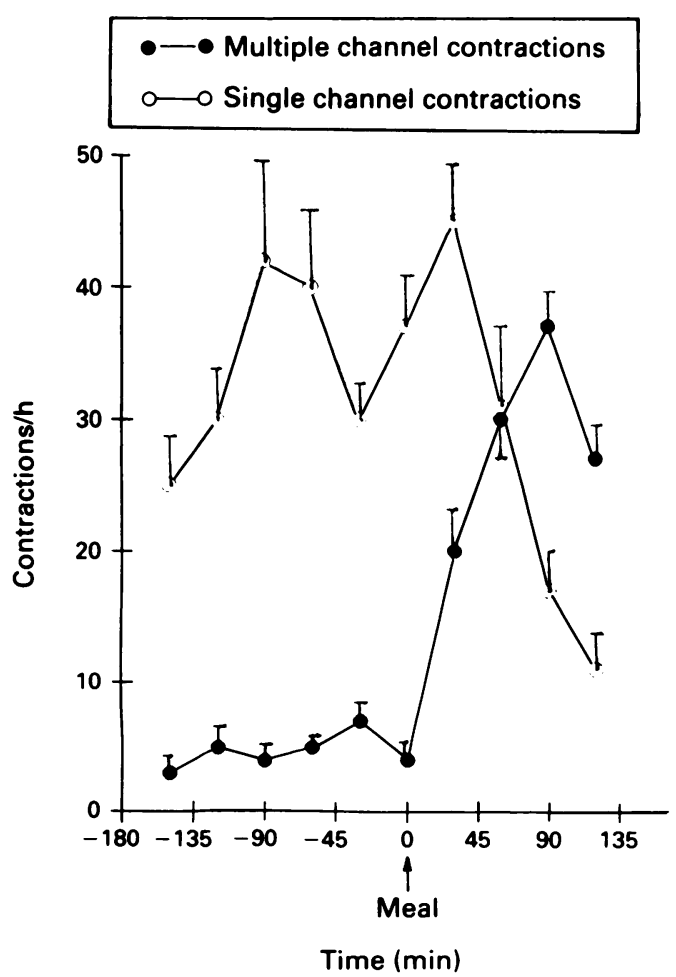

Figure 3: The mean number of single and multiple channel contractions before and after a meal in study 1 . 
Within 15 minutes of ingesting the meal, there was an increase in the motility index, which was composed of an increase in multiple channel contractions. It does seem unlikely that these simultaneous pressure waves were caused by large contractions at one site transmitted through the common cavity of the rectosigmoid. If this were a common cavity phenomenon, then why were single channel pressure waves also recorded at the same time? There was also no evidence of a serial delay in the upstroke of the pressure wave in sequential channels that would be indicative of propagation.

Therefore, we conclude that these multiple channel contractions represent simultaneous non-propagating contractions similar to those seen in the distal colon after a meal by MorenoOsset and his colleagues. ${ }^{7}$ Loening-Baucke ${ }^{8}$ has reported the occurrence of what she termed synchronous and non-synchronous contractions and showed that the former were more common postprandially. This postprandial pattern was, however, not associated with any increase in the motility index.

Local infusion of mebeverine hydrochloride directly into the sigmoid colon changed the rectosigmoid response to a meal by stopping the rise in the multiple channel contractions. Mebeverine had no significant effect on the motility index or on the incidence of single channel contractions. A positive drug effect may have been overlooked if independent analysis of the types of contraction had not been done.

The significance of multiple channel contrac- tions lies in their association with rectal symptoms. Seventy five per cent of our subjects experienced rectal symptoms only in association with a multiple channel contractions and the prevention of these contractions with mebeverine also removed symptoms.

In conclusion, our results show that the rectosigmoid response to a meal is associated with a considerable increase in the rate of one type of contraction and a reduction in the rate of another. Simply counting numbers of contractions or calculating motility indices may mask significant changes in rectosigmoid motility patterns, which may be of use in identifying symptom generating events and assessing the response to drugs.

1 Kock NG, Hulten L, Leandor L. A study of motility of different parts of the human colon. Resting activity, respons to feeding and to prostigimine. Scand $\mathcal{f}$ Gastroenterol 1968; 3 : 163-9.

2 Sun WM, Edwards CA, Prior A, Rao SSC, Read NW. Effect of oral nicardipine on anorectal function in normal human oral nicardipine Dis Sci 1990; 35: 885-90.

3 Tomlin J, Brown S, Cann PA, Read NW. Is rectosigmoid colonic response to food modulated by proximal colonic colonic response to food modulated by

4 Dinoso VP, Goldstein J, Rosner B. Basal motor activity of the distal colon. A reappraisal. Gastroenterology 1983; 85: 637-42.

5 Chowdhury AR, Dinoso VP, Lorber SH. Characterization of a hyperactive segment at the rectosigmoid junction. Gastroenterology 1976; 71: 584-8.

6 Narducci F, Bassotti G, Gaburri M, Morelli A. Twenty-fou hour manometric recording of colonic motor activity in healthy man. Gut 1987; 28: 17-25.

7 Moreno-Osset E, Bazzocchi G, Lo S, Trombley B, Ristow E Reddy SN, et al. Association between postprandial changes in colonic intraluminal pressure and transit. Gastroenterology 1989; 96: 1265-73.

8 Loening-Baucke V, Anuras S. Effect of a meal on the mobility of the sigmoid colon and rectum in healthy adults. $A m \mathcal{F}$ Gastroenterol 1983; 78: 393-7. 